\title{
Quality in Use Model for Web Portals (QiUWeP)
}

\author{
Mayte Herrera, Ma Ángeles Moraga, Ismael Caballero, and Coral Calero \\ Alarcos Research Group, Department of Technologies and Information Systems, \\ University of Castilla - La Mancha, \\ Paseo de la Universidad 4, 13071, Ciudad Real, Spain \\ mayteherreranoa@gmail.com, \\ \{MariaAngeles.Moraga, Ismael.Caballero, Coral.Calero\} @uclm.es
}

\begin{abstract}
Web Portals are increasingly being used for important tasks, at a work, personal or leisure related issues. Since Web Portals provide with applications, services and information, their levels of quality are an important prerequisite for their success, and hence, a way to ensure that they do not disappear as a consequence of their not having been used. According to the ISO, there are various perspectives of quality: internal, external, and in use. Although issues related to external quality and internal quality have been widely studied in literature, we consider that it is also necessary to take account the importance of quality in use of web portals. This paper is aimed at defining a quality model to assess the level of quality in use of Web Portals. The model is founded on the ISO/IEC 25010 standard, and on some related works found in literature.
\end{abstract}

Keywords: web portal, quality in use, quality model, quality characteristics.

\section{Introduction}

Web Portals appeared in the late 1990s as a new type of Internet website architecture specifically designed to provide personalized online services [1]. The success of Web portals depends on their ability to provide accurate content and useful services specifically tailored to individual users according to their requirements [1]. Web portals offer a single point of access to a wide range of information, applications and services in a single environment [2], thus enabling and facilitating the collaboration and interaction between users [3] in such a way that all the services could be adapted to the preferences or necessities of each user [4]. Since web portals allow a suitable work environment to be created for any organization [5], companies establish and promote their web portals to complement, replace or expand their services to their customers, and may even have the intention of providing new services to new customers.

Several researchers have highlighted the importance of developing Web portals which could successfully attract new users and maintain existing ones by serving as a gateway to information and internet services [6]. One important success factor is, therefore, the need to warranty the levels of quality of the web portals as software products [7]. It is thus no longer sufficient to simply provide technically excellent web portals, but it is also necessary for them to fit the best practices and activities of their consumers [8]. 
As with any other kind of software product, ISO defines several perspectives with which to analyze the level of quality of a web portal: internal and external quality, which are related to the characteristics or properties of the portal, and quality in use, which is aimed at evaluating users' opinions of the portal.

Unfortunately, the relevant literature has not dealt with quality in use in web portals in sufficient depth, despite its having been proved to be an important aspect [1]. In the domain of the web portals specifically, if users do not feel safe when using a portal, if it is difficult for them to achieve their goals through the Web portal, or if they are not satisfied when using it, they may easily decide to use another different one and the web portal could consequently disappear. In the context of organizational web portals, workers are the final users. If the web portal does not fit their specific tasks, it could be very difficult for them to achieve their goals. Assessing the quality in use will allow web portal owners to estimate how usable a web portal might be and the user's satisfaction.

To assess quality in use, it is first necessary to define a model. The main contribution of this paper is, therefore, to define a quality in use model for web portals taking the ISO/IEC 25010 as a basis. ISO/IEC 25010 is the new standard of software product quality that is awaiting publication, and is a part of the new series of SQuaRE (Software product Quality Requirements and Evaluation) standards [9].

This paper is structured as follows. Related literature is presented and analyzed in Section 2. This section is divided into two subsections, the first of which is related to the perspectives of quality in SQuaRE, and is specifically focused on the quality in use model, and the characteristics and sub-characteristics proposed in the standard [9]. The second subsection analyzes certain works that study quality in use in web portals. In section 3 a discussion about the topics and the proposal of a model for quality in use for web portals based on ISO/IEC 25010 is presented. Finally, Section 4 shows some of our conclusions and proposed future work.

\section{Related Literature}

This section reviews certain concepts related to the perspective of quality in software. More specifically, we shall focus on the perspective of quality in use provided by the ISO/IEC 25010 standard. We shall also analyze how the concept of quality in use in the context of web portals has been studied in literature, along with the set of characteristics that affect quality in the use of portals.

\subsection{Perspective of Quality in SQuaRE}

The ISO/IEC 9126 standard [10] for software product quality has recently been superseded by the new ISO/IEC 25000 series: the Software product Quality Requirements and Evaluation (SQuaRE) set of standards [9]. According to both standards, the quality of a system can be assessed as the extent to which the system satisfies the stated and implied needs of its various stakeholders. These stated and implied needs are represented in the SQuaRE by means of different models: the software product quality model, the data quality model and the system quality in use model. 
The main purpose of the software product quality model is to specify and assess the level of quality of a product through internal measures of inherent properties of the software, and through external measures of the behavior of the system of which the software is part [9].

External quality can be assessed as the result of the combination of the behavior of both the software and the computer system [8], whereas quality in use is the extent to which a product which is being used by specific users meets their needs to achieve specific goals with effectiveness, efficiency, flexibility, safety and satisfaction in specific contexts of use [9]. Quality in use therefore corresponds to users' views of the quality of a system containing software. In this respect, the perception of quality in use must be measured in terms of the result of using the software, rather than the properties of the software itself [8]. In order to make the assessment process easier, the quality in use model defined in ISO/IEC 25010 [9] defines three main characteristics: usability, safety and flexibility.

The characteristic of usability has been defined [9] as the extent to which a product can be used by specified users to achieve specified goals with effectiveness, efficiency and satisfaction in a specified context of use. The users' goals may be pragmatic (to be effective and efficient), and/or hedonic (to achieve stimulation, identification and/or evocation). The pragmatic goals are: acceptable perceived experience of use (pragmatic aspects including efficiency), acceptable perceived results of use (including effectiveness), and acceptable perceived consequences of use (including safety). The hedonic and pragmatic satisfaction in this model is achieved through likability, pleasure, comfort and trust. User performance and satisfaction is determined by qualities including attractiveness, functional suitability and ease of use [9].

The characteristic of safety has been inherited from ISO/IEC 9126-1 [10]. It is defined as the degree of expected impact of harm to people, to businesses, to data, to software, to property or to the environment in the intended contexts of use [11]. Safety could be broadly interpreted as the capability to measure the potentially negative outcomes which could be generated from incomplete or incorrect input [11]. For a consumer of a product, negative business consequences may not only be associated with poor performance, but also, for example, with the lack of pleasurable emotional reactions, or of achievement of other hedonic goals [11]. Safety has the following sub-characteristics: commercial damage, operator health and safety, public health and safety, and environmental harm.

The need to consider the context has been made explicit in the new definition of quality in use in ISO/IEC CD 25010 [11]. This is owing to the importance of considering that a product which is usable in one context of use may not be usable in another context with different users, tasks or environments. A new characteristic of flexibility has therefore been included, with the sub-characteristics of context conformity, context extendibility and accessibility [9]. Flexibility also includes aspects related to the users' ability to modify their means of interaction with other users, and the appearance of the web portals' interface to suit their individual needs and preferences [9].

The quality in use model defined by ISO/IEC 25010 is shown in Fig. 1, and a complete definition of the quality characteristics and sub-characteristics can be found in [9]. 


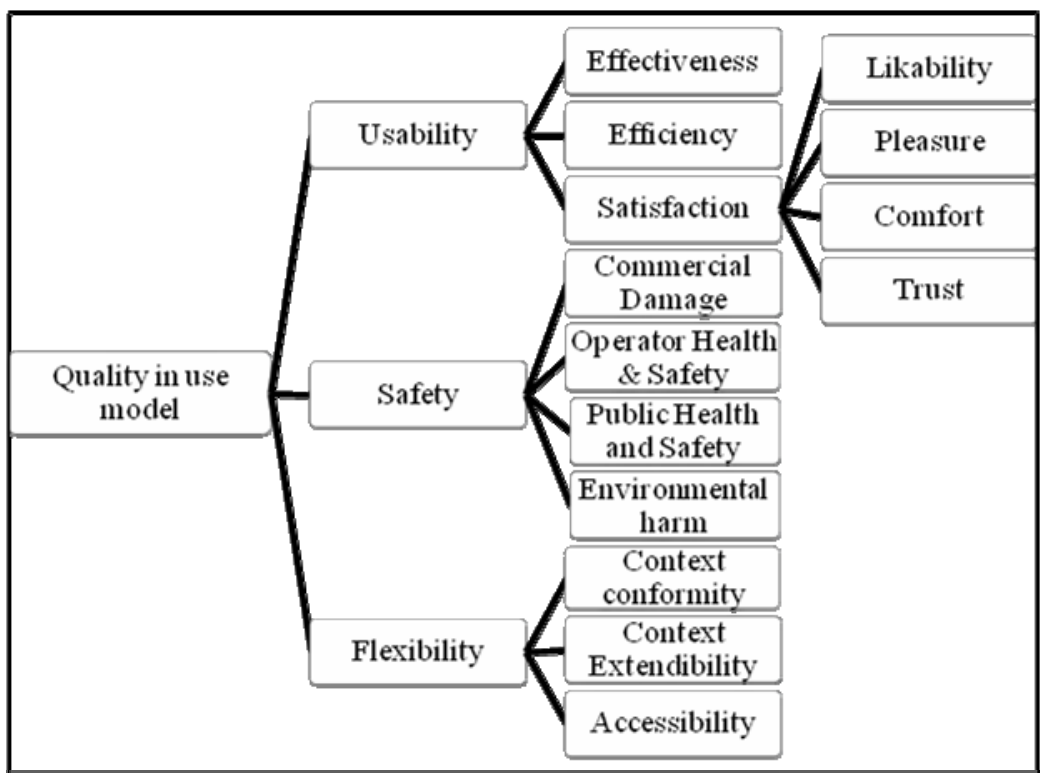

Fig. 1. Model for quality in use in ISO/IEC 25010

\subsection{Quality in Use in Web Portals}

As was previously mentioned, ISO/IEC 25010 is an evolution of the ISO/IEC 9126 and defines a more complete and detailed quality in use model. Nevertheless, some characteristics regarding quality in use have already been studied in previous works and will be analyzed in this section. The main aim of this section is to study how the characteristics and sub-characteristics for quality in use have been defined in recent studies. This will be done in order to adapt the quality in use model defined in ISO/IEC 25010 [9] to the context of Web portals and to complete the model proposed in this study. It is important to emphasize the fact that the quality characteristics to be analyzed are those concerning the quality in use and those that are of interest to the end users of web portals. These characteristics directly evaluate the effects on users as a result of using the portal, and the effectiveness and efficiency in carrying out their tasks using it. With this in mind, it is not the interest to this study, to analyze aspects or characteristics of the web portals themselves, as these are features of external quality.

The characteristic of Satisfaction has been considered as an element of quality for web applications in general, and has already been introduced in the area of web engineering [12]. The work presented in [6] specifically studied user satisfaction with regard to the usage of web portals. This study outlined the initial results of how a multi-criteria evaluation framework was applied to synthesize the multiple dimensions related to users' satisfaction with a web portal. In their work, the authors studied user satisfaction from the point of view of [6]:

- the web portal content, in terms of contained information, or when accessing external information resources. 
- the web portal design, in terms of providing users with a pleasant, usable and stable environment,

- the web portal personalization capabilities, in terms of serving users' specific preferences and needs,

- the web portal support for the formulation of virtual communities of users, in terms of bringing together users with similar interests and needs.

WebQual[13] is an instrument for assessing characteristics such as usability, information quality, and service interaction quality. The current version of WebQual (4.0) has been renamed as E-qual. This version is based on the analysis of the results of its previous versions complemented by an extensive discussion of the literature that supports each dimension. The main change is an increased emphasis on usability rather than site quality. The focus of attention is on user experience when using a Web Portal rather than site characteristics per se [2]. The dimensions included in E-Qual are:

- usability, which encompasses aspects such as navigation, appearance and general ease of use,

- information quality, which encompasses accuracy, timeliness, relevance, granularity and general believability of the information,

- service interaction quality, which encompasses service quality constructs such as security, trust, personalization and access to the organization.

Works of $[1,2,7,14,15]$ analyzes the perceived quality of service in web portals. It is necessary to point out that this term refers to portal users' perceptions of the quality of all the information, applications and services supplied. These studies point out that ensuring that these services meet quality requirements is essential to ensure business operations and user satisfaction [2].

The works presented in [14] and in [15], both based on E-Qual, study the perceived portal quality. The factors that were confirmed in these studies are similar to those of the previous studies on which they are based, with slight differences in terminology and emphasis that will be shown as follows. It is important to specify that in some studies the term "factor" is used with the same meaning as that of "quality characteristics".

In [15] the authors carry out an empirical study of organizational portals. This proposal identifies four factors of service quality:

- empathy, which is similar to service interaction quality in E-Qual,

- ease of use, which is similar to usability in E-Qual,

- information quality, which maintains the same name as that used in E-Qual,

- accessibility, which is similar to aspects of usability and service interaction quality in E-Qual.

Similarly, in[14] a study of business-to-consumer portals in Greece identified three factors for service quality in this type of portals:

- customer care and risk reduction benefit, which is similar to service interaction quality in E-Qual,

- information benefit, which is similar to information quality in E-Qual,

- Interaction facilitation benefit, which is similar to usability in E-Qual, but extended by adding considerations of technical design and speed. 
In [1] the authors have made a study of the perceived quality of service in a University Web portal. The authors initially based their study on E-Qual, and then added some other factors that they considered might be significant for web portal users. They therefore propose to extend their understanding of user perceived e-service quality to include transaction quality, and they include some additional items hypothesized from the literature. Transaction quality includes aspects of complete transactions that are useful for the portal users.

Finally, it is important to consider the study of [16]. In this work a revised list of characteristics was developed into an Internet based questionnaire for measuring user satisfaction with web sites. Questions were placed in different groups. These groups or factors were [16]:

- Ease of use, which is related to the ability to easily navigate through a site and find required information. Important aspects include simple, intuitive and consistent navigation,

- Experience, which includes the visual and personal experience of visiting the site. Issues include design, use of colors and style, along with building interest and a sense of community,

- Information, which is aimed at accessing content with an adequate levels of quality information. This information is appropriate for consumption by the user, and should typically be easy to read and understand, relevant, current, reliable and provided via an appropriate level of detail and format,

- Communication and integration, which are related to the way in which the site is integrated with the external environment and communication with the user. This includes being able to find and return to a site, integration or links with other sites, the speed and security of communication, and provision for feedback and other contact.

\section{Proposal for a Quality in Use Model for Web Portals (QiUWeP)}

This section provides a definition of a model for quality in use for web portals. In order to achieve this goal, and as a starting point, the quality characteristics and subcharacteristics defined in the quality in use model defined by the ISO/IEC 25010 standard [9] have been analyzed and selected.

The standard defines usability, safety and flexibility. Since all of these characteristics affect the use of web portals by final users, they were adapted to the web portal context. Some sub-characteristics defined in the standard, which will be specified later, were adapted to the contexts of web portals. However, other sub-characteristics were not included because they could be considered as not being sufficiently relevant for web portal usage. The obtained model was modified as a result of the analysis of the related existing works shown in the previous section. What follows is an analysis of the characteristics and sub-characteristics found in the quality in use model of the ISO/IEC 25010 standard and in the proposals found in literature.

The definition of the characteristic of usability was taken from the standard [9] and adapted to the domain of web portals. Usability has the sub-characteristics of "effectiveness", "efficiency" and "satisfaction" as reported in the standard [9]. The effectiveness sub-characteristic is defined in terms of accuracy and completeness of the 
achievement of the users' goals. The efficiency sub-characteristic is defined in terms of the resources used by portal users as they carry out their tasks by using the portal. With regard to the satisfaction sub-characteristic, the works found that study important aspects for web portal users' satisfaction were $[1,2,6,12,15]$. These works have been used as the basis to define the sub-characteristics of satisfaction. As a result of this analysis of the related literature, the sub-characteristics of satisfaction which were added to the model were: the ease with which users can perform their tasks through the portal, the users' visual and personal experiences, the perceived interaction quality, the perceived transaction quality, and finally the sense of being in community while using a portal. These sub-characteristics will be analyzed in detail as follows.

The easier it is to use a portal, the more satisfied the users will be. This signifies that the portal should be easy to navigate, easy to learn how to operate, and it should be easy to find information/resources that users' need. "Experience" is a subcharacteristic that includes the user's visual and personal experience as a result of using a web portal. This sub-characteristic includes the following aspects: the effect of the design of the web portal on the users' satisfaction, along with the perception of acceptable response times, the sense of being in control while doing their tasks, and the attractiveness and enjoyability for users. The "quality of the interactions perceived" includes aspects related to portal users' interactions with the organization through its web portal. Some important aspects of the interaction quality are the ease of communication with the organization through its portal, and the ease of managing and integrating the roles and relationships that users have with the organization. The "perceived transaction quality" sub-characteristic has been supported by some previous studies to include aspects that assess the users' satisfaction with a web portal as a result of completing all the transactions or operations they expect and need to achieve their goal. Another sub-characteristic of satisfaction is "sense of community", which can be defined in terms of user satisfaction with regard to the feeling of being part of a group of users with similar interests and needs.

The definition of safety characteristic was borrowed from the standard [9] but renamed as security. From the perspective of final users of web portals, the sub-characteristics defined for security are, therefore, "personal security risk" and "economic damage risk". These new sub-characteristics are being proposed for the first time in this paper, and they are part of the contribution of this work. They were identified as a result of analyzing the web portal context, and the definition of the safety characteristic and its sub-characteristics in the ISO/IEC 25010 [9] standard. The safety sub-characteristics proposed by the standard that were the basis of the new sub-characteristics defined in our proposal were: "Commercial damage" and, "Public health and safety". Commercial damage is defined in the standard as the degree of expected impact of harm to commercial property, operations or reputation in the intended contexts of use. This could include costs of correcting erroneous output, inability to provide an acceptable service that could affect users directly, or loss of current or future sales. Public health and safety are defined according to the ISO/IEC 25010 standard [9] as being the degree of expected impact of harm to the public in the intended contexts of use. The complete definition of the security sub-characteristics will be specified in the proposal for the model below.

Flexibility, when referring to web portals, this indicates whether the portal can be used by a large number of users with different preferences or cultures, or even different 
levels of disabilities. Flexibility must therefore be taken into account in the context of portals, and we therefore decided to adapt it and to add it to our proposal. "Accessibility" and "personalization" have been identified as being the sub-characteristics of flexibility. Accessibility was proposed and defined by the standard [9] as the degree of usability for users with specified disabilities. This definition was used in reference to the ISO 9241-171 definition: "the usability of a product, service, environment or facility by people with the widest range of capabilities" [17]. The personalization sub-characteristic was not defined as a sub-characteristic in the ISO/IEC 25010 standard, but is clearly mentioned in the context of use of final users, and is also supported by previous studies.

One important aspect that deserves to be mentioned is the importance of data and information quality in a web portal environment. As we have seen in the related literature, data and information quality are important prerequisites for overall quality in use [18]. People who use data originating from portals in, for example, their work need to be sure that the data has an adequate level of quality. Although the importance of data and information quality has been proven, they will not be included in this proposal owing to the fact that SQuaRE clearly differentiates between the quality of a product and the quality of the data manipulated by the software product. Moreover, the authors of [19] have defined a data quality model for web portals, denominated as SPDQM (SQuaRE Portal Data Quality Model), which is the reference that can be used to assess the level of data and information quality in a web portal.

Table 1 shows the characteristics and sub-characteristics defined in this proposal as a result of the aforementioned analysis. This table gathers together the related studies that support the identification of each of these characteristics and sub-characteristics that are part of the quality in use model for web portals.

Table 1. Characteristics and sub-characteristics supported by related literature

\begin{tabular}{|c|c|c|c|c|c|c|c|c|c|}
\hline \multicolumn{10}{|c|}{ Quality in use for web portals characteristics and subcharacteristics } \\
\hline \multicolumn{3}{|l|}{ QiUWeP } & [9] & [6] & [13] & [14] & [15] & [1] & [16] \\
\hline \multirow{7}{*}{ Usability } & \multicolumn{2}{|c|}{ Effectiveness } & $\mathrm{x}$ & & & & & & \\
\hline & \multicolumn{2}{|c|}{ Efficiency } & $\mathrm{x}$ & & & & & & \\
\hline & \multirow{5}{*}{ 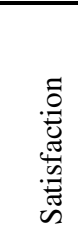 } & Ease of Use & & & $\mathrm{x}$ & & $\mathrm{x}$ & & $\mathrm{x}$ \\
\hline & & Experience & & & & & & & $\mathrm{x}$ \\
\hline & & Perceived Interaction Quality & & & $\mathrm{x}$ & $\mathrm{x}$ & $\mathrm{x}$ & & $\mathrm{x}$ \\
\hline & & Perceived Transaction Quality & & & & & & $\mathrm{x}$ & \\
\hline & & Sense of Community & & $\mathrm{x}$ & & & & & $\mathrm{x}$ \\
\hline \multirow{2}{*}{ Security } & \multicolumn{2}{|c|}{ Personal Security Risk } & $\mathrm{x}$ & & & $\mathrm{x}$ & & & \\
\hline & \multicolumn{2}{|c|}{ Economic damage risk } & $\mathrm{x}$ & & & $\mathrm{x}$ & & & \\
\hline \multirow{2}{*}{ Flexibility } & \multicolumn{2}{|c|}{ Accessibility } & $\mathrm{x}$ & & & & $\mathrm{x}$ & & \\
\hline & \multicolumn{2}{|c|}{ Personalization } & & $\mathrm{x}$ & & & & & \\
\hline
\end{tabular}


The quality in use model for web portals proposed in this study are specified below, where all the definitions of the characteristics and sub-characteristics of the model have been defined. The quality in use model for web portals is shown in Fig. 2 .

1. Usability: The extent to which a web portal can be used by specific users to achieve specified goals with effectiveness, efficiency and satisfaction in a specified context of use.

1.1. Effectiveness: The degree to which the web portal can be used by the users to achieve their specific goals with accuracy and completeness in a specified context of use.

1.2. Efficiency: The degree of resources consumed by web portal users in relation to the effectiveness reached in a specific context of use.

1.3. Satisfaction: the web portal users' degree of satisfaction with regard to achieving their pragmatic and hedonic goals in a given context of use. These goals are related to likability, trust and pleasure.

1.3.1. Ease of Use: The web portal users' degree of satisfaction in relation to being able to use the portal easily to achieve their goals. Important aspects include simple, intuitive and consistent navigation.

1.3.2. Experience: The web portal users' degree of satisfaction in relation to the visual and personal experience of visiting the portal. Issues include design and style, enjoyability or entertainment, along with acceptable response times for users/clients.

1.3.3. Perceived Interaction Quality: The web portal users' degree of satisfaction with regard to the experience of interacting with the organization via its web portal.

1.3.4. Perceived Transaction Quality: The web portal users' degree of satisfaction with regard to being able to carry out and complete all the operations that they wish to through the web portal.

1.3.5. Sense of Community: The web portal users' degree of satisfaction in relation to meeting, collaborating and communicating with other users with similar interests and needs.

2. Secutiry: The degree, to which the web portal does not, under specified conditions, lead to a state in which the personal security of its users is endangered and economic damage is caused.

2.1. Personal Security Risk: the degree of expected impact of harm to the personal security of the portal's users or clients in the intended contexts of use.

2.2. Economic damage risk: the degree of expected impact of causing economic damage to the web portal users owing to insecure operations in the intended contexts of use.

3. Flexibility: the degree to which the quality in use requirements for web portals can be achieved in different contexts of use and for as many users as possible. Flexibility can be achieved by adapting the web portal to additional user groups or cultures. Flexibility enables portals to consider circumstances, opportunities and individual preferences.

3.1. Accessibility: the degree of effectiveness, efficiency, safety and satisfaction, when people with the widest range of capabilities use the web portal.

3.2. Personalization: the degree to which the users can modify certain aspects of the portal to suit their specific preferences and needs. 


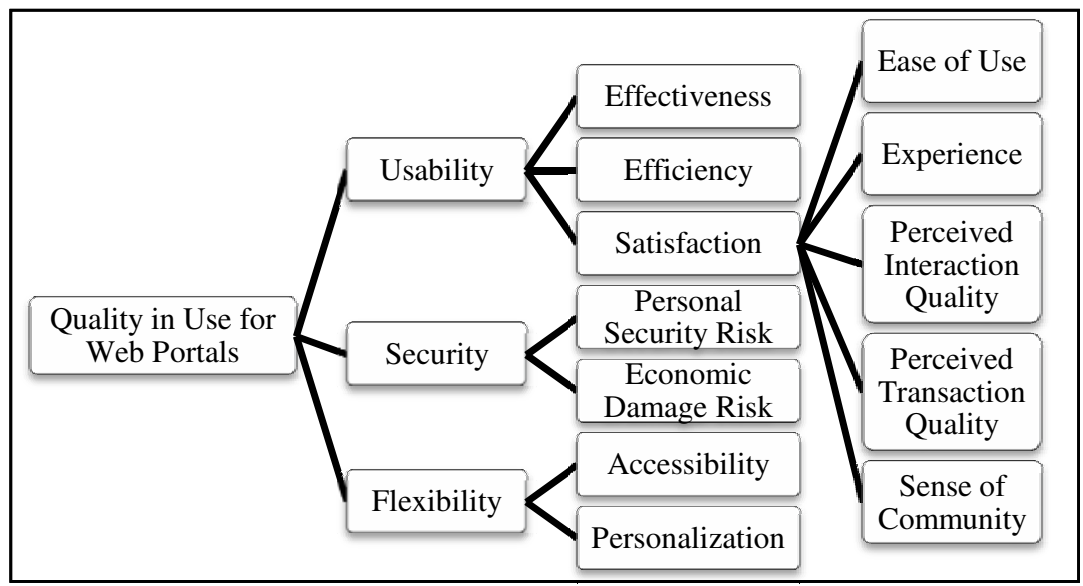

Fig. 2. Quality in use model for Web Portals

\section{Conclusions and Future Work}

As the contents of this paper show, the quality of web portals is worth observing and should be evaluated taking into account the different perspectives of quality. In this respect, the quality in use is the perception of the quality provided by final users, and the results of using the portal. Although some characteristics of quality in use have been studied for the context of web portals in the literature analyzed, no quality in use model is aligned with the ISO/IEC 25010 standard. In this proposal we have defined a quality in use model for web portals, based on the ISO/IEC 25010, the new standard for software product quality and quality in use. This approach takes into account the portal users' perspective and therefore aims to assess the results of use of the web portal, both from the perspective of user satisfaction, and the effectiveness and efficiency of the users while they use the web portal. Our proposal can be used to assess the quality perceived when using a web portal and achieving user's goals. This proposal takes as basis the ISO/IEC 25010 standard [9], since this is an evolution of ISO/IEC 9126, along with certain related previous studies which were taken into account in order to define the characteristics and sub-characteristics of the model that are needed to assess quality in use in the context of web portals. As part of this work we are currently defining measures of effectiveness, efficiency, security and flexibility. For these measurements, we are working on a questionnaire to assess the satisfaction of the users of a web portal. As part of our future work, the appropriateness of this model will be validated by applying it to assess the quality in use of various web portals in different context of use. This will be made using techniques of empirical validations as surveys and the analysis of the results of these evaluations will make possible refine the model if it were necessary. 


\section{Acknowledgments}

This research is part of the projects PEGASO-MAGO (TIN2009-13718-C02-01), and DQNet (TIN2008-04951-E/TIN), both supported by the Spanish Ministerio de Educación y Ciencia, and TALES (HITO-2009-14), both supported by the Consejería de Educación y Ciencia of Junta de Comunidades de Castilla-La Mancha.

\section{References}

1. Tate, M., et al.: Stakeholder Expectations of Service Quality in a University Web Portal, p. 1-21 (2009)

2. Tate, M., Evermann, J., Hope, B., Barnes, S.: Perceived Service Quality in a University Web Portal: Revising the E-Qual Instrument (2007)

3. Aneja, A., Brooksby, B., Rowan, C.: Corporate portal framework for transforming content chaos on intranets. Intel Technology Journal 11, 21-28 (2001)

4. Ángeles Moraga, M., Calero, C., Piattini, M.: A First Proposal of a Portal Quality Model. In: IADIS International Conference (2004)

5. Collins, H.: Corporate Portal Definitions and Features. Amacom Books, New York (2001)

6. Manouselis, N., Sampson, D.G.: Multiple Dimensions of User Satisfaction as Quality Criteria for Web Portals. In: ICWI 2004, pp. 535-542. IADIS (2004)

7. Liu, C.-T., Du, T.C., Tsai, H.-H.: A study of the service quality of general portals. Information \& Management 46(1), 52-56 (2009)

8. Bevan, N.: Quality in use: Meeting user needs for quality. Journal of Systems and Software 49, 89-96 (1999)

9. ISO/IEC CD 25010.3: Systems and software engineering - Software product Quality Requirements and Evaluation (SQuaRE) - Software product quality and system quality in use models. ISO (2009)

10. ISO/IEC 9126-1: Software engineering - Product quality - Part 1: Quality model. ISO (2001)

11. Bevan, N.: Extending Quality in Use to Provide a Framework for Usability Measurement, pp. 13-22 (2009)

12. Zhang, P., Small, R.V., Dran, G.: Websites that Satisfy Users: A Theoretical Framework for Web User Interface Design and Evaluation. In: Proceedings of 32nd IEEE International Conference on System Sciences, Hawaii, USA (1999)

13. WebQual home page, http: / / www . webqual.co.uk/

14. Gounaris, S., Dimitriadis, S.: Assessing service quality on the web: Evidence from business to consumer portals. The Journal of Services Marketing 17, 529 (2003)

15. Kuo, T., Lu, I.-Y., Huang, C., et al.: Measuring user's perceived portal service quality: An empirical study. Total Quality Management and Business Excellence 16, 309 (2005)

16. Barnes, S., Vidgen, R.: WebQual: An exploration of Web-site Quality. In: Proceedings of the Eighth European Conference on Information Systems, Vienna (2000)

17. ISO 9241-171: Ergonomics of human-system interaction - Part 171: Guidance on software accessibility. ISO (2008)

18. Wynn, M., Zhang, S.: Web Portals in SMEs - Two Case Studies. In: Proc. of the 2008 Third International Conference on Internet and Web Applications and Service (ICIW). IEEE Computer Society, Los Alamitos (2008)

19. Carmen Moraga, M.M., Calero, C., Caro, A.: SQuaRE-Aligned Data Quality Model for Web Portals. In: Ninth International Conference on Quality Software, pp. 117-122 (2009) 